\title{
sciendo
}

\section{Isokinetic Strength of Rotators, Flexors and Hip Extensors is Strongly Related to Front Kick Dynamics in Military Professionals}

\author{
by \\ Michal Vagner ${ }^{1}$, Jan Malecek ${ }^{1}$, Luboš Tomšovský2, Petr Kubový2, Andrea Levitova², \\ Petr Stastny
}

\begin{abstract}
Achieving the maximum possible impact force of the front kick can be related to the isokinetic lower limb muscle strength. Therefore, we aimed to determine the regression model between kicking performance and the isokinetic peak net moment of hip rotators, flexors, and hip extensors and flexors at various speeds of contraction. Twenty-five male soldiers $(27.7 \pm 7.2 \mathrm{yrs}, 83.8 \pm 6.1 \mathrm{~kg}, 180.5 \pm 6.5 \mathrm{~cm})$ performed six barefoot front kicks, where impact forces $(\mathrm{N})$ and kick velocity $\left(\mathrm{m} \cdot \mathrm{s}^{-1}\right)$ were measured. The 3D kinematics and isokinetic dynamometry were used to estimate the kick velocity, isokinetic moment of kicking lower limb hip flexors and extensors $\left(60,120,240,300^{\circ} \cdot \mathrm{s}^{-1}\right)$, and stance lower limb hip internal and external rotators $\left(30,90^{\circ} \cdot \mathrm{s}^{-1}\right)$. Multiple regression showed that a separate component of the peak moment concentric hip flexion and extension of the kicking lower limb at $90^{\circ} \cdot \mathrm{s}^{-1}$ can explain $54 \%$ of the peak kicking impact force variance $\left(R^{2}=0.54 ; p<0.001\right)$. When adding the other 3 components of eccentric and concentric hip internal and external rotations at $30^{\circ} \cdot \mathrm{s}^{-1}$, the internal and external hip rotation ratios at $30^{\circ} \cdot \mathrm{s}^{-1}$ on the stance limb and the concentric ratio of kicking limb flexion and extension at $300^{\circ} \cdot \mathrm{s}^{-1}$ that explained the variance of impact force were $75 \%(p=0.003)$. The explosive strength of kicking limb hip flexors and extensors is the main condition constraint for kicking performance. The maximum strength of stance limb internal and external rotators and speed strength of kicking limb hip flexors and extensors are important constraints of kicking performance that should be considered to improve the front kick efficiency.
\end{abstract}

Key words: strike, self-defense, impact force, peak moment, resistance training.

\section{Introduction}

The self-defense of civilians and military personnel includes tactical and technical skills, where the front kick is the most common strike used in close combat (Vágner et al., 2018). Particularly, military personnel use this movement pattern because they often carry

equipment in their hands and are wearing backpacks. To eliminate the attacker, the goal of a front kick is to achieve the maximum possible impact force ( $\left(\mathrm{IF}_{\text {peak }}\right)$ when the foot hits the target, requiring strong and fast muscle action of the lower limb. However, there is limited information

\footnotetext{
1 - Charles University in Prague, Faculty of Physical Education and Sport, Department of Military, Jose Martiho 31, post. 16252 Prague, Czech Republic.

2 - Charles University, Faculty of Physical Education and Sport, department of Anatomy and Biomechanics, Prague, Jose Martiho 31, ZIP 162 52, Czech Republic.

3 - Charles University, Faculty of Physical Education and Sport, department of Adapted Physical Education and Sport Medicine, Prague, Jose Martiho 31, ZIP 162 52, Czech Republic.

4 - Charles University in Prague, Faculty of Physical Education and Sport, Department of Sport Games, Jose Martiho 31, post. 162 52 Prague, Czech Republic.
} 
regarding which muscle groups should be trained to improve the efficiency of the front kick.

An efficient front kick is related to the technique, movement control, speed, velocity, force, energy and power (Maszczyk et al., 2018; Ramakrishnan et al., 2018; Serina and Lieu, 1991; Vágner et al., 2018), where those components are determined by the quantity of transferred momentum or energy (Dworak et al., 2008). Thus, many researchers have investigated the kinetics and kinematics of front kicks (Mori et al., 2002; Pozo et al., 2011; Wasik et al., 2015). The techniques of the front kick begin from the center of the body by flexion in the hip and knee joints and forward-kicking leg thigh acceleration. Furthermore, the movement of the thigh slows down and the knee joint accelerates the movement of the foot in the direction of the impact (Sorensen et al., 1996). In the analysis of the individual parts of the front kick, the highest torque was observed in the hip joint flexors (Hipflex) in the first phase of the kick; however, in the second phase, the hip extensor (Hipext) and knee extensors dominated. The largest moment was observed about the hip joint with the next highest being produced about the knee joint (Hwang, 2008). Despite detailed descriptions of the front kick technique, no study has investigated the relationship of kicking performance to hip joint rotation (Hiprot), which is the important part of other types of kicks (Sujae and Koh, 2010) and might be an important contributor to front kicking efficiency.

The muscle groups, which are tested for the isokinetic net moment output, are selected according to their contribution to specific movement tasks, e.g., Hipflex, Hipext, knee extensors and abductors have been found to be important during front kicking (Busko, 2016; Jung et al., 2017), where different combat sport athletes showed similar peak Hipflex and Hipext moment values (Busko, 2016). Although the front kick primarily comprises fast muscle action, no study has focused on whether maximum, explosive or speed strength determines the IF $\mathrm{F}_{\text {peak }}$ in a front kick. This may be done by isokinetic measurement at different speeds and types of contraction (Stastny et al., 2018), where low, medium and high speed indicate the athletes' maximum strength, explosive strength and speed strength, respectively. In addition to Hipflex and
Hipext of the kicking limb, the Hiprot moment of the stance limb might be useful to describe a soldier's ability to perform front kicking because their strength has been related to dynamic stability (Gordon et al., 2013). To our best knowledge, the current literature does not summarize how large is the muscle contribution of a stance and a kicking lower limb to front kicking performance and what type of the force/velocity relationship should be applied for valid isokinetic strength testing in subjects trained in front kicking.

Achieving the maximum possible impact force of the front kick can be related to the isokinetic lower limb muscle strength, where there are no current guidelines regarding which muscle groups and testing velocities are crucial for kicking performance. Therefore, we aimed to determine the regression model between kicking performance (represented by the total force impulse, $V_{\text {imp }}$ and $I_{\text {peak }}$ ) and isokinetic peak net moment of internal Hiprot and external Hiprot Hipflex, and Hipext at various speeds of contraction $\left(30^{\circ} \cdot \mathrm{s}^{-1}, 60^{\circ} \cdot \mathrm{s}^{-1}, 90^{\circ} \cdot \mathrm{s}^{-1}, 120^{\circ} \cdot \mathrm{s}^{-1}, 240^{\circ} \cdot \mathrm{s}^{-1}\right.$ and $\left.300^{\circ} \cdot \mathrm{s}^{-1}\right)$.

\section{Methods}

The present investigation was a crosssectional study performed at the Biomechanics Laboratory of Extreme Loading at the Charles University in Prague, Faculty of Physical Education during a special military forces selfdefense camp (February, 2017). Following a familiarization session, participants reported to the laboratory and performed two series of six maximal effort front kicks against a vertically anchored force plate, followed by one day of rest and isokinetic strength measurement. The participant performed all front kicks barefoot by their dominant lower limb, which was estimated by asking about the preference of soccer and combat kicking. The research was approved by the Ethics Committee of the Faculty of Physical Education and Sport (No. 50/2018); furthermore, informed consent was signed by all the participants. The procedures were performed in accordance with the Declaration of Helsinki 2013.

All front kick dynamics measurements were completed in approximately $40 \mathrm{~min}$. The dynamic warm-up lasted $10 \mathrm{~min}$, and each participant performed a pretest of five kicks in the power plate before the experimental 
measurement. The familiarization session was also used to measure the distance from the force plate for each participant to execute each kick in the same, comfortable position during testing. These individualized distances were then recorded and used to ensure the same starting position for each kick. All front kicks began with a front posture and were executed so that the foot made contact at a mid-range height, typically the abdomen or solar plexus (Vágner et al., 2018). Participants executed two sets of six front kicks, with instructions to kick as fast and as hard as possible to the target (force plate). Between each kick, the participant was given $30 \mathrm{~s}$ rest intervals (Vagner et al., 2018), and there was a 5-min rest interval between particular sets of kicks.

\section{Participants}

Twenty-five professional soldiers (age: $27.7 \pm 7.2$ yrs, body mass: $83.8 \pm 6.1 \mathrm{~kg}$, body height: $180.5 \pm 6.5 \mathrm{~cm}$ ) from the Military Department at the Faculty of Physical Education and Sport, Charles University in Prague participated in the experiment. Participants were familiarized twice with the testing protocol before the study began and were instructed not to perform any physically demanding activities three days prior to testing. All soldiers were healthy for the duration of the experiment and did not suffer from any disease during the study period. The inclusion criteria were front kicking training for at least 2 years, the absence of muscle soreness, the absence of any musculoskeletal injury for 2 months prior to the study and 2 years of special forces training. All the participants were informed of the testing protocols and all aspects of the study before they provided written informed consent. Additionally, written informed consent was obtained from participants pictured in Figure 1 for the publication of their images.

\section{Dynamics of the front kick}

The kinetics of each front kick was measured using a triaxial force plate (Kistler 9281; Winterthur, Switzerland), recording at a sampling rate of $1000 \mathrm{~Hz}$. In the preanalytical phase, the lower limit of the magnitude of the acting force was set at $100 \mathrm{~N}$ to reduce noise on the force plate and mark the beginning and end of the interaction between a participant's foot and the force plate. The plate was adjustable along the vertical axis to ensure that the height of the plate was individualized to each participant's "mid-range" height (Dworak et al., 2008; Kuragano and Yokokura, 2012; Vágner et al., 2018) for the purposes of the experiment (Figure 1).

The peak impact force ( $\left.\mathrm{IF}_{\text {peak }}\right)$ was determined as the maximum value of the sum of force exerted in all three directions $\mathrm{x}, \mathrm{y}, \mathrm{z}$ (Dworak et al., 2008) (Eq. 1, Figure 1). Figure 1 shows an example of the force-time curve for a single front kick (Ramakrishnan et al., 2018).

$\left|\overrightarrow{F_{\text {peak }}}\right|=\max \left(\sqrt{\overrightarrow{F_{x}^{2}}+\overrightarrow{F_{y}^{2}}+\overrightarrow{F_{z}^{2}}}\right)$

The time to peak force in each front kick was analyzed from the first $20 \mathrm{~N}$ interaction of a participant's foot with the force plate $\left(t_{0}\right)$ to reaching the peak force $\left(t_{\text {peak }}\right)$ (Eq. 2$)$.

$\left(t_{\text {max }}\right)=\left(t_{\text {peak }}\right)-\left(t_{0}\right)$

The impulse of the impact force acting during the whole time $\left(t_{\max }\right)$ of a front kick was derived from the general formula for the impulse (Eq. 3) and was modified for the discrete data measured by the force plate. For each time period $(\Delta t)$, defined by the sampling frequency, the impulse was calculated. By summing them, the overall net impulse of a front kick was determined (Eq. 4) (Vágner et al., 2018).

$\vec{I}=\int_{t_{0}}^{t} \vec{F} d t$

$\overrightarrow{I_{\text {net }}}=\sum_{i=1}^{n}(\vec{F})_{i} \cdot \Delta t_{i}$

\section{Velocity of the front kick}

Three-dimensional kinematic data were collected using a six-camera motion analysis system (Qualisys 2.2, Sweden) set up to sampling frequency of $500 \mathrm{~Hz}$. Velocity data were collected from retro-reflective markers placed on the participant's malleolus lateralis to represent the foot velocity, which was gathered from the peak marker velocity before the impact initiation on the force plate. The force plate and 3D motion capture data were fully synchronized and collected using Qualisys software 2.2 (Qualisys Track Manager, Sweden). The impact velocity ( $\mathrm{V}_{\mathrm{imp}}$ ) was gathered from the lateral malleolus marker from the $10 \mathrm{~ms}$ time frame prior to the first recorded $2 \mathrm{~N}$ impact to the force plate.

\section{Isokinetic strength testing}

All isokinetic strength tests were performed using a standard dynamometer (Humac Norm; CSMi Stoughton, MA, USA), where the peak moments of the Hipflex and Hipext of the kicking dominant limb and internal and 
external Hiprot of the non-dominant lower limb (stance limb during kicking) were measured at different angular velocities (Stastny et al., 2018). In addition to the peak force measurement, the antagonist ratios were calculated from the values of the peak moments. Calibration of the unit was performed at the beginning of each test day according to the 502140 HUMAC NORM guidelines.

Hipflex and Hipext were tested in the supine position with the dynamometer lever arm aligned with the axis of the femur great trochanter at the angular velocities of $30^{\circ} \cdot \mathrm{s}^{-1}, 120^{\circ} \cdot \mathrm{s}^{-1}, 240^{\circ} \cdot \mathrm{s}^{-1}$ and $300^{\circ} \cdot \mathrm{s}^{-1}$. For each velocity, the participant performed six antagonist consecutive repetitions of maximum voluntary contraction in concentric muscle action, with a $90 \mathrm{~s}$ rest interval between the tested velocities. The movement of Hipflex and Hipext was accomplished within the possibility of the individual range of motion of each participant with a minimum range of $120^{\circ}$ of Hipflex.

The internal and external Hiprot were tested in the supine position with the extended knee and the hip joint at angular velocities of $30^{\circ} \cdot \mathrm{s}^{-1}$ and $90^{\circ} \cdot \mathrm{s}^{-1}$. Each participant performed three repetitions of the maximum voluntary contraction at concentric and eccentric muscle action in two sets. The dynamometer was set up to perform concentric internal and external HipRot in antagonist action, followed by eccentric internal and external Hiprot, where each maximal effort was followed by a $90 \mathrm{~s}$ rest interval. Movement of the internal and external Hip Rot was accomplished within the possibility of the individual range of motion of each participant with a minimum range of $35^{\circ}$ of the internal and external Hiprot. The ratios between concentric contractions were called conventional ratios and those between eccentric and concentric actions were called functional ratios.

\section{Statistics}

All statistical analyses were performed using NCSS version 2004 (Number Cruncher Statistical Systems, Kaysville, Utah) with the alpha level set at 0.01 . The mean and standard deviation of all six kicks and all isokinetic measurements were calculated along with the intraclass correlation coefficient across performed repetitions (ICC). The Shapiro-Wilk and Kolmogorov-Smirnov tests were used to determine whether the data were normally distributed. In the event of normal distribution, the Pearson's product moment correlation coefficient was used to examine the relationships between the isokinetic data vs. velocity and impact force of front kicks. The reliability of the isokinetic peak torque, kick velocity and impact force variables were estimated by the intraclass correlation coefficient (ICC).

First, a simple linear regression was created, followed by multiple regression and the construction of a regression model. The isokinetic data that significantly correlated $(p<0.01)$ with the front kick performance measurements were used in the basic regression model as predictors to construct a linear regression model to determine the $\mathrm{IF}_{\text {peak. }}$ Furthermore, we used isokinetic data that were not significantly correlated with the $\mathrm{IF}_{\text {peak }}$ to extend the basic regression model.

\section{Results}

The average impulse force of the front kick was $2202 \pm 489 \mathrm{~N}$, and average velocity of the front kick was $7.7 \pm 1.03 \mathrm{~m} \cdot \mathrm{s}^{-1}$, where all the repeated measurements showed high reliability (ICC above 0.90) and data normality was not disrupted (Table 1). However, most highly correlated values were observed between $V_{\text {imp }}$ and internal Hiprot concentric and eccentric $90^{\circ} \mathrm{S}$ $(\mathrm{r}=0.22, p=0.28$ and $0.36, p=0.08)$, internal/external Hiprot conventional ratio $30 \cdot \mathrm{s}(\mathrm{r}=0.28, p=0.18)$ and internal/external functional Hiprot $30^{\circ} \cdot \mathrm{s} \quad(\mathrm{r}=0.22$, $p=0.30$ ), but without significance. Additionally, no correlation was found between $V_{\text {imp }}$ and Hipflex or Hipext concentric and the $\mathrm{IF}_{\text {peak. }}$.

Hip rotation (stance lower limb)

No significant correlation was observed between Hiprot and the IF peak of the front kick. The correlation revealed high values between the $\mathrm{IF}_{\text {peak }}$ of the frontal kick and External Hiprot Ecc $90^{\circ} \mathrm{s}$ $(\mathrm{r}=0.31, p=0.13)$, Internal Hiprot Ecc $90^{\circ} \cdot \mathrm{s}(\mathrm{r}=0.21$, $p=0.30)$, but both were not significant. Additionally, no relationship was found between the $\mathrm{IF}_{\text {peak }}$ of the front kick vs. the internal/external conventional and internal Ecc/external functional Hiprot peak torque.

Hip flexion and extension (kicking lower limb)

A significant correlation was observed between the IF peak of the front kick and Hipflex and Hipext moment. The high correlation was observed between the $\mathrm{IF}_{\text {peak }}$ and Hipflex concentric at $120^{\circ} \cdot \mathrm{s}(\mathrm{r}$ $=0.64, p<0.001)$ and Hipext concentric 
$120^{\circ} \cdot \mathrm{s}(\mathrm{r}=0.72, p<0.001)$. A moderate correlation was found between the $\mathrm{IF}_{\text {peak }}$ and concentric Hipext at $30^{\circ} \cdot \mathrm{s}, 240^{\circ} \cdot \mathrm{s}$ and $300^{\circ} \cdot \mathrm{s}(\mathrm{r}=0.53,0.61,0.59$, respectively, $p<0.001)$. Since the Hipflex and Hipext concentric at $120^{\circ} \cdot \mathrm{s}$ showed the highest values, this component was used as the first factor in a further regression model. Linear regression showed a relationship between the $\mathrm{IF}_{\text {peak }}$ and concentric Hipflex at $120^{\circ} \cdot \mathrm{s}\left(\mathrm{R}^{2}=0.41 ; p<0.001\right.$, Figure 2) and Hipext at $120^{\circ} \cdot \mathrm{s}\left(\mathrm{R}^{2}=0.51 ; p<0.001\right.$, Figure 2). When a linear regression model was constructed using these two variables as one component to the predicted IF peak of the front kick, the predictive power of the model was $54 \%$ of the variability $\left(\mathrm{R}^{2}=0.54 ; p<0.001\right)$ (Figures 2 and 4 ).

Figure 3 shows that participants who achieved high levels of Hipflex and Hipext and had more weight achieved a better IF $I_{\text {peak. The }}$ correlation coefficients between the body mass of participants vs. Hipflex and Hipext moment were $r=$ $0.54(p=0.005)$ and $\mathrm{r}=0.38(p=0.06)$, respectively. Additionally, the correlation coefficient between the body mass of participants vs. IF peak was $\mathrm{r}=0.46$ $(p=0.02)$. Therefore, we calculated the relative values of the IF peak per $\mathrm{kg}$ of body mass. The correlation coefficient between the IF $\mathrm{IFeak}_{\text {and }}$ a relative $\mathrm{IF}_{\text {peak }}$ per $\mathrm{kg}$ of body mass was $\mathrm{r}=0.94(p=$ 0.001 ). When body mass was used as a partial variable, the relationship between the $\mathrm{IF}_{\text {peak }}$ and isokinetic variables was decreased by $8 \%$.

Hip flexion, extension and rotation (kicking and standing leg)

The regression model using concentric Hipflex and Hipext $120^{\circ} \cdot \mathrm{s}$ as a separate component combined with the component of concentric and eccentric Hip ${ }_{\text {Rot }}$ Con at $30^{\circ} \cdot \mathrm{s}^{-1}$ increased the $\mathrm{IF}_{\text {peak }}$ prediction by $12 \% \quad\left(\mathrm{R}^{2}=0.66 ; \quad p=0.0016\right)$. Additionally, when we added to this component the model of Internal/external conventional Hiprot at $30^{\circ} \cdot \mathrm{s}^{-1}$ and internal eccentric/external concentric functional Hiprot at $30^{\circ} \cdot \mathrm{s}^{-1}$, the prediction of the $\mathrm{IF}_{\text {peak }}$ of the front kick was $71 \%$ of the variability $\left(\mathrm{R}^{2}=0.71 ; p=0.0033\right)$. The last variable component that we could add to the regression model was the conventional ratio between Hipflex $/$ Hipext at $300^{\circ} \cdot \mathrm{s}^{-}$ 1 , which increased the $\mathrm{IF}_{\text {peak }}$ prediction by $4 \%\left(\mathrm{R}^{2}=\right.$ $0.75 ; \quad p=0.0031)$. This model comprised independent variables that were mentioned above predicted $75 \%$ of the variability in the IF peak of the front kick (Figure 4).

\section{Discussion}

Although various techniques, conditions and tactical aspects may be related to the front kick IF $_{\text {peak, }}$ our study showed that the isokinetic strength of hip flexion and extension are strong physical components predicting the amount of $\mathrm{IF}_{\text {peak, }}$ which can explain more than half of its value. Moreover, this finding indicates that not maximal strength, but explosive strength and speed strength of hip flexors and extensors should be the target of kick-specific testing and training. On the other hand, our study did not include knee extensors and flexors as in some previous studies (Busko, 2016; Ramakrishnan et al., 2018) and may be added in future studies to design an even more complex and stronger regression model. However, the principal goal of isokinetic testing is to use a rather smaller amount of the testing task, but with high validity for the tested population purposes. Based on the high regression of our first independent component of hip flexion and extension at $120^{\circ} \cdot \mathrm{s}^{-1}$, we can conclude that testing only those two muscle groups may provide valid information about the physical ability to perform the front kick with a high $\mathrm{IF}_{\text {peak }}$ in highly trained soldiers.

Our regression model has four components, where $17 \%$ of the variance is explained by the maximum concentric and eccentric strength of hip external and internal rotation regarding the stance of the lower limb. This result supports a practical approach of the front kick technique, where stance limb rotation should highly contribute to the beginning of a kick and should support the whole body during the foot impact. Therefore, we may conclude that hip rotation typical for round kicking (Sujae and Koh, 2010) should also be beneficial in front kick training. 
Table 1

Isokinetic net moment during hip external rotation, internal rotation, hip flexion, extension and their strength ratios at different movement speeds

\begin{tabular}{|c|c|c|c|c|c|}
\hline Peak moment $(\mathrm{N} \cdot \mathrm{m})$ & Average \pm SD & CI lower & CI upper & SW & KS \\
\hline External Hip Rotation Con $30^{\circ} \cdot \mathrm{s}$ & $68 \pm 17$ & 61 & 75 & 0.97 & 0.08 \\
\hline Internal Hip Rotation Con $30^{\circ} \cdot \mathrm{s}$ & $57 \pm 11$ & 52 & 61 & 0.97 & 0.09 \\
\hline External Hip Rotation Con $90^{\circ} \mathrm{s}$ & $65 \pm 16$ & 58 & 71 & 0.98 & 0.10 \\
\hline Internal Hip Rotation Con $90^{\circ} \cdot \mathrm{s}$ & $52 \pm 10$ & 48 & 56 & 0.98 & 0.13 \\
\hline External Hip Rotation Ecc $30^{\circ} \cdot \mathrm{s}$ & $83 \pm 23$ & 73 & 92 & 0.90 & 0.21 \\
\hline Internal Hip Rotation Ecc $30^{\circ} \cdot \mathrm{s}$ & $60 \pm 16$ & 53 & 67 & 0.72 & 0.20 \\
\hline External Hip Rotation Ecc $90^{\circ} \cdot \mathrm{s}$ & $81 \pm 25$ & 71 & 92 & 0.88 & 0.24 \\
\hline Internal Hip Rotation Ecc $90^{\circ} \cdot \mathrm{s}$ & $61 \pm 10$ & 57 & 66 & 0.96 & 0.13 \\
\hline Internal/external Conv $90^{\circ} \cdot \mathrm{s}$ & $0.82 \pm 0.16$ & 0.76 & 0.89 & 0.95 & 0.12 \\
\hline Internal/external Conv $30^{\circ} \cdot \mathrm{s}$ & $0.85 \pm 0.17$ & 0.79 & 0.92 & 0.95 & 0.14 \\
\hline Internal ecc/external Functional $90^{\circ} \cdot \mathrm{s}$ & $0.99 \pm 0.23$ & 0.90 & 1.09 & 0.97 & 0.10 \\
\hline Internal ecc/external Functional $30^{\circ} \cdot \mathrm{s}$ & $0.91 \pm 0.23$ & 0.82 & 1.00 & 0.96 & 0.10 \\
\hline Hip flexion Con $30^{\circ} \cdot \mathrm{s}$ & $186 \pm 31$ & 173 & 199 & 0.98 & 0.12 \\
\hline Hip extension Con $30^{\circ} \cdot \mathrm{s}$ & $362 \pm 78$ & 329 & 132 & 0.95 & 0.14 \\
\hline Hip flexion Con $120^{\circ} \cdot \mathrm{s}$ & $143 \pm 27$ & 132 & 155 & 0.96 & 0.11 \\
\hline Hip extension Con $120^{\circ} \cdot \mathrm{s}$ & $281 \pm 57$ & 257 & 304 & 0.96 & 0.14 \\
\hline Hip flexion Con $240^{\circ} \cdot \mathrm{s}$ & $109 \pm 23$ & 99 & 118 & 0.97 & 0.09 \\
\hline Hip extension Con $240^{\circ} \cdot \mathrm{s}$ & $217 \pm 56$ & 194 & 240 & 0.97 & 0.07 \\
\hline Hip flexion Con $300^{\circ} \cdot \mathrm{s}$ & $94 \pm 22$ & 85 & 103 & 0.97 & 0.08 \\
\hline Hip extension Con $300^{\circ} \cdot \mathrm{s}$ & $208 \pm 54$ & 186 & 230 & 0.96 & 0.08 \\
\hline Hip flexion/extension $30^{\circ} \cdot \mathrm{s}$ & $0.53 \pm 0.09$ & 0.37 & 0.72 & 0.97 & 0.11 \\
\hline Hip flexion/extension $120^{\circ} \cdot \mathrm{s}$ & $0.52 \pm 0.07$ & 0.39 & 0.69 & 0.98 & 0.11 \\
\hline Hip flexion/extension $240^{\circ} \cdot \mathrm{s}$ & $0.52 \pm 0.11$ & 0.35 & 0.82 & 0.94 & 0.12 \\
\hline Hip flexion/extension $300^{\circ} \cdot \mathrm{s}$ & $0.47 \pm 0.11$ & 0.29 & 0.78 & 0.92 & 0.15 \\
\hline
\end{tabular}

Con = concentric, $E C C=$ eccentric, $S D=$ standard deviation, $S W=$ Shapiro-Wilk test, $K S=$ Kolmogorov-Smirnov test,$C I=$ confidence interval, $C o n v=$ conventional strength ratio. 


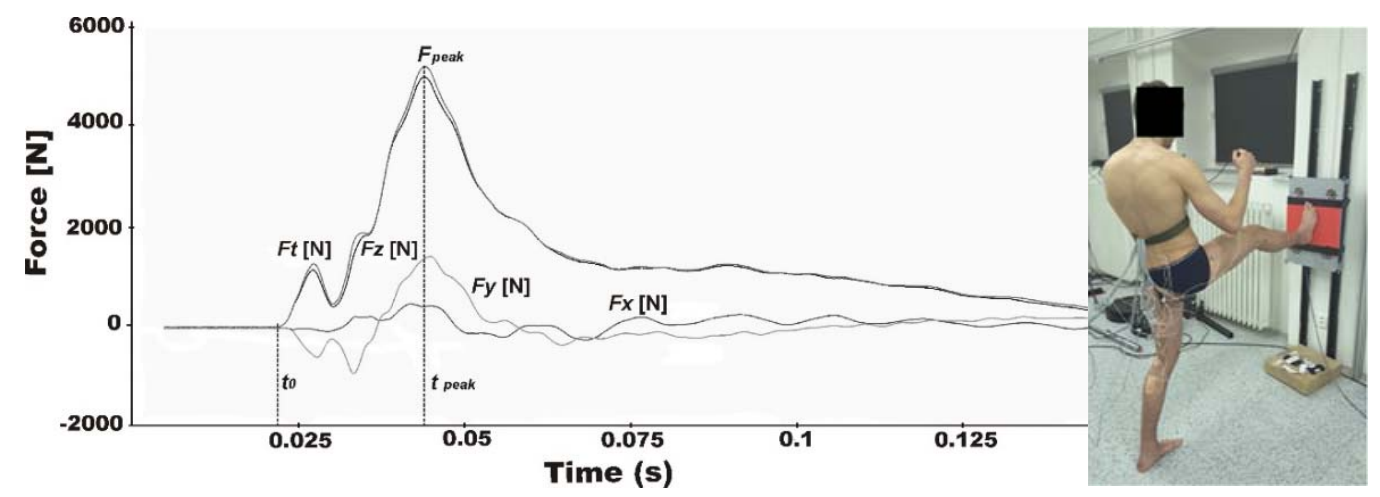

Figure 1

A participant performing the front kick to a vertically anchored force plate and the time course of the kinetic force recorded during the kick.
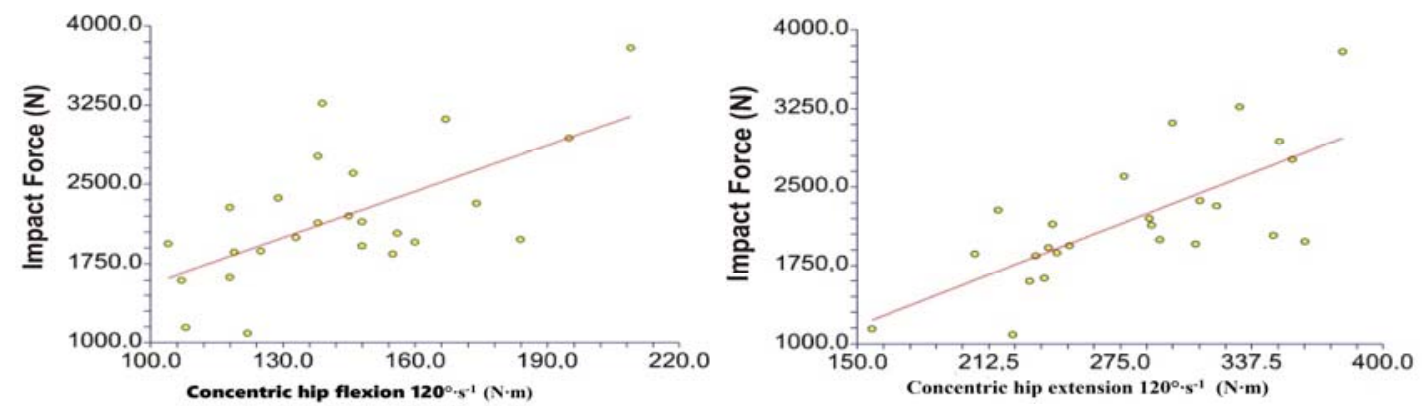

Figure 2

Regression of the impact force of the front kick vs. concentric hip extension at $120^{\circ} \cdot \mathrm{s}$ and vs. concentric hip flexion at $120^{\circ} \cdot \mathrm{s}^{-1}$ 

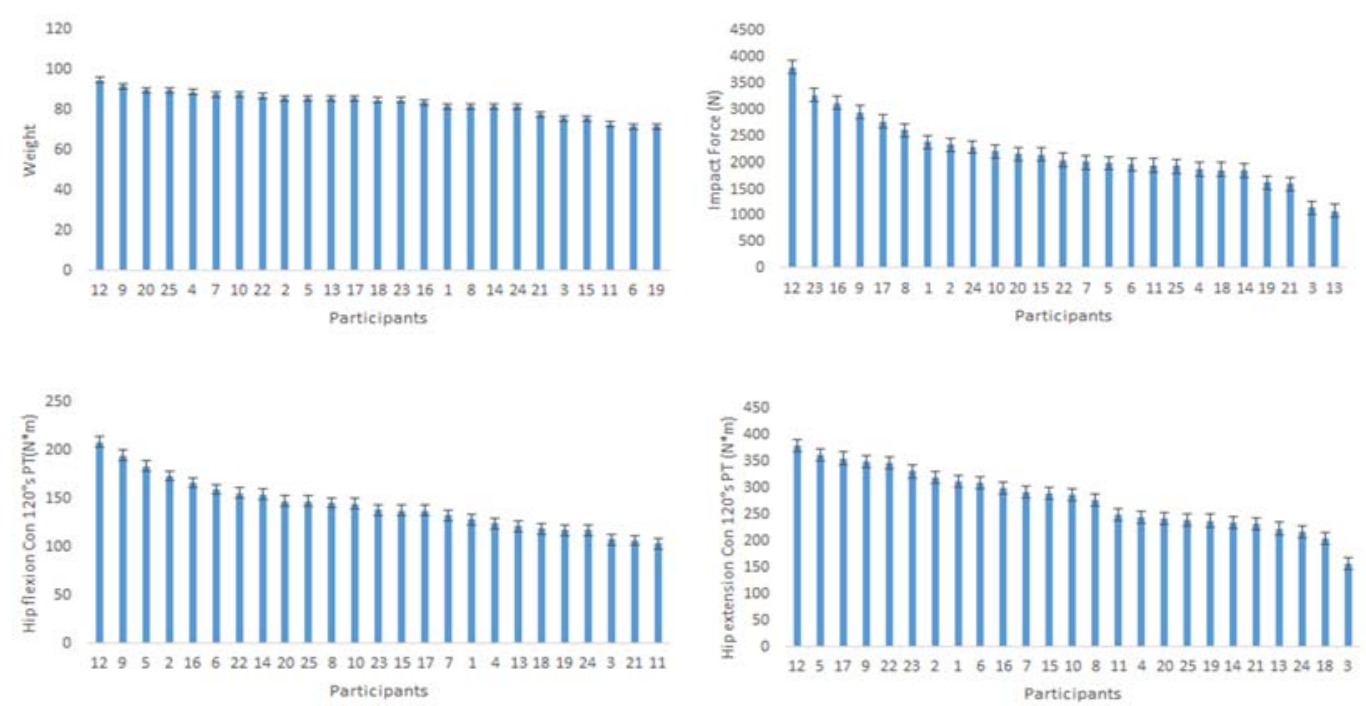

Figure 3

Con hip extension $120^{\circ} \cdot \mathrm{s}$, con hip flexion $120^{\circ} \cdot \mathrm{s}$, body mass and impact force delivered by individual participants with error bars showing the maximum and minimum values.

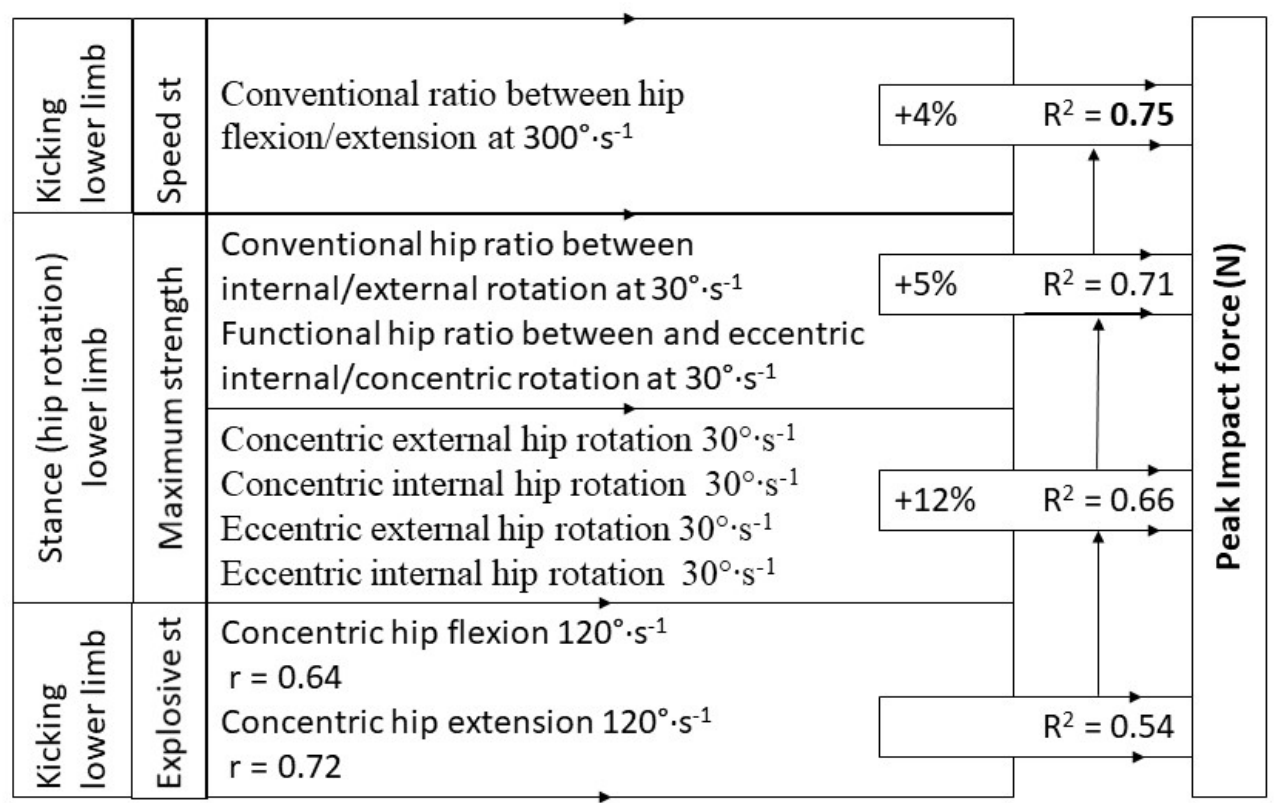

Figure 4

Composite regression model from isokinetic variable predictors. St $=$ strength 
The finding that the hip rotation ratio and eccentric rotator strength appear in our model supports that those muscles contribute when kicking is accelerated, but also when kinetic energy is being transferred to the target. On the other hand, our study found a correlation between body mass and the $\mathrm{IF}_{\text {peak, while a }}$ previous study between body mass and energy transfer to the target (Ramakrishnan, et al., 2018) regardless of training levels of kickers. This suggests that the body mass is an important aspect of the kicking IF $_{\text {peak. The effect of body }}$ mass on the increase in the stroke force was found in correlation studies (Estevan et al., 2011; Pędzich et al., 2006). However, this fact did not reduce significantly our regression model, which was composed of isokinetic variables. We believe that if the soldier's body can keep a high level of stability on the stance leg during the kick, the probability of transfer energy to the target increases.

The isometric strength of Hipflex and Hipext is documented in combat sports (boxing, judo, taekwondo), where the average Hipflex $98 \mathrm{Nm}$ and Hipext 427 Nm (Busko, 2016) have been reported. Values of Hipflex $(186 \pm 31 \mathrm{Nm})$ and Hipext $(362 \pm 78$ $\mathrm{Nm})$ at $30^{\circ} \cdot \mathrm{s}-1$ obtained in the present study are not comparable due to the differences in the isokinetic protocol and a different study population. However, we can state that our military professionals had more balanced Hipflex/Hipext strength ratios than combat sport athletes, which may refer to their complex strength development of the lower limb antagonist groups. Previous studies assume the high importance of the isokinetic knee flexion moment, knee extension moment thigh adductors and thigh adductors moment (Jung et al., 2017; Probst et al., 2007) for kicking performance in karate and taekwondo athletes. Our study did not include these muscle groups based on the assumption that strength of the knee extension and flexion cannot be fully applied in complex movement without sufficient strength development of Hipflex and Hipext. With regard to hip adductors and abductors, we preferred a deeper muscle group of hip rotators, of which function is directly responsible for whole-kick stability. Our results showed that the hip internal and external strength ratio is an independent component in our model, where our participants had a functional ratio as high as $0.91 \pm 0.23$. This supports our hypothesis that not just individual muscle strength, but also antagonist balance plays an important role in kicking efficiency. Although our regression model resulted in a high amount of the explained variable, we still recommend estimating the role of knee extensors and flexors in future studies.

Since no current normative data exist about the $\mathrm{IF}_{\text {peak, }}$ we cannot categorize our average value of $2.2 \pm 0.5 \mathrm{kN}$ to a performance level. However, our data are in the range of previously reported $\mathrm{IF}_{\text {peak }}$ values of $1.17-7.79 \mathrm{kN}$, where athletes or soldiers from different martial arts reported average values of $3.89 \mathrm{kN}$ (Ramakrishnan et al., 2018), 2.9 kN (Dworak et al., 2008), and $3.4 \mathrm{kN}$ (Vágner et al., 2018). The average $V_{\text {imp }}$ of the front kick was $7.7 \pm 1.03 \mathrm{~m} \cdot \mathrm{s}^{-1}$. Nevertheless, no correlation was found between $V_{\text {imp }}$ and isokinetic variables or IF peak, a finding that is similar to a study performed on soccer players (Mognoni et al., 1994). Therefore, we can conclude that the level of isokinetic strength of the muscle is not related to the velocity of the kick.

Our results should be used in military personnel and combat sport training, where we recommend exercising antagonist muscle groups and hip stabilizers (Stastny et al., 2016), preferably in a unilateral fashion (Stastny et al., 2015a, 2015b) and regulating the exercise tempo during the eccentric muscle action (Wilk et al., 2018a, 2018b). The hip flexors and extensors should be progressively trained for maximum strength, followed by explosive strength development and at the final stages of conditioning programs for speed strength, to improve the efficiency of the front kick. The internal and external hip rotators should be trained in unilateral and antagonist exercises focusing on maximum strength and eccentric muscle action, to improve the efficiency of the front kick.

One limitation of the present study is that the setup of the testing procedure was not identical to the environment of outdoor practices, but simulated well-prepared, kick-adjusted (optimal) and self-started front kicking action. Moreover, our regression model did not include a range of hip motion, the strength of all lower limb muscle groups, the kick accuracy and any perceptional requirements. 


\section{Conclusions}

The explosive strength of kicking limb hip flexors and extensors is the main condition constraint for kicking performance. Therefore, exercises enhancing explosive strength should be included in programs focused on front kicking. Maximum strength of the stance limb internal and external rotators and speed strength of kicking limb hip flexors and extensors are important constraints of kicking performance and should be considered in improving the front kick. The external and internal rotators should be trained by concentric and eccentric contractions with equal strength of both antagonists.

\section{Acknowledgements}

This study was a part of a research project UNCE/HUM/032 at Charles University in Prague.

\section{References}

Busko K. Comparison of Muscle Strength in Male Combat Sport Athletes. Polish Journal of Sport and Tourism, 2016; 23(4): 186-189

Dworak LB, Dziewiecki K, Maczynski J. Characteristics of kinematics and kinetics of strokes in karatebiomechanical approach. In, ISBS-Conference Proceedings Archive; 2008

Estevan I, Álvarez O, Falco C, Molina-García J, Castillo I. Impact force and time analysis influenced by execution distance in a roundhouse kick to the head in taekwondo. J Strength Condit Res, 2011; 25(10): 2851-2856

Gordon AT, Ambegaonkar JP, Caswell SV. Relationships between core strength, hip external rotator muscle strength, and star excursion balance test performance in female lacrosse players. Int. J Sports Phys Ther, 2013; 8(2): 97

Hwang I. Analysis of the kicking leg in taekwondo. In, ISBS-Conference Proceedings Archive. 2008

Jung HC, Lee S, Seo MW, Song JK. Isokinetic assessment of agonist and antagonist strength ratios in collegiate taekwondo athletes: A preliminary study. Sport Sciences for Health, 2017; 13(1): 175-181

Kuragano T, Yokokura S. Experimental Analysis of Japanese Martial Art Nihon-Kempo. ICHPER-SD J ReS, 2012; 7(1): 40-45

Maszczyk A, Gołaś A, Pietraszewski P, Kowalczyk M, Cięszczyk P, Kochanowicz A, Smółka W, Zając A. Neurofeedback for the enhancement of dynamic balance of judokas. Biol Sport., 2018; 35(1): 99-102

Mognoni PM, Narici V, Sirtori MD, Lorenzelli F. Isokinetic torques and kicking maximal ball velocity in young soccer players. J Sports Med Phys Fitness, 1994; 34(4): 357-361

Mori S, Ohtani Y, Imanaka K. Reaction times and anticipatory skills of karate athletes. Hum Mov Sci, 2002; 21(2): 213-230

Pędzich W, Mastalerz A, Urbanik C. The comparison of the dynamics of selected leg strokes in taekwondo WTF. Acta of Bioengineering and Biomechanics, 2006; 8(1): 83-90

Pozo J, Bastien G, Dierick F. Execution time, kinetics, and kinematics of the mae-geri kick: comparison of national and international standard karate athletes. J. Sports Sci., 2011; 29(14): 1553-1561

Probst MM, Fletcher R, Seelig DS. A comparison of lower-body flexibility, strength, and knee stability between karate athletes and active controls. J Strength Condit Res, 2007; 21(2): 451

Ramakrishnan KR, Wang H, Shankar K, Fien A. A new method for the measurement and analysis of biomechanical energy delivered by kicking. Sports Eng., 2018; 21(1): 53-62

Serina E, Lieu D. Thoracic injury potential of basic competition taekwondo kicks. J Biomech., 1991; 24(10): 951-960

Sorensen H, Zacho M, Simonsen EB, Dyhre-Poulsen P, Klausen K. Dynamics of the martial arts high front kick. J Sports Sci., 1996; 14(6): 483-495

Stastny P, Lehnert M, Tufano JJ. Muscle Imbalances: Testing and Training Functional Eccentric Hamstring Strength in Athletic Populations. JoVE, 2018; 135: e57508

Stastny P, Lehnert M, Zaatar AM, Svoboda Z, Xaverova Z. Does the Dumbbell-Carrying Position Change the Muscle Activity in Split Squats and Walking Lunges? J Strength Condit Res, 2015a; 29(11): 3177-3187 
Stastny P, Tufano JJ, Golas A, Petr M. Strengthening the Gluteus Medius Using Various Bodyweight and Resistance Exercises. Strength Condit J, 2016; 38(3): 91-101

Stastny P, Tufano JJ, Lehnert M, Golas A, Zaatar A, Xaverova Z, Maszczyk A. Hip abductors and thigh muscles strength ratios and their relation to electromyography amplitude during split squat and walking lunge exercises. Acta Gymnica, 2015b; 45(2): 51-59

Sujae IH, Koh MT. Joint Moment Contributions to Non-Planar Kicking Techniques: Insights into Kuda and Sila Serve Mechanics. International Journal of Sports Science \& Coaching, 2010; 5(1): 47-60

Vagner M, Thiel D, Jelen K, Tomsovsky L, Kubovy P, Tufano JJ. Wearing ballistic and weighted vests increases front kick forces. Archives of Budo, 2018; 14: 231-237

Vágner M, Tomšovský L, Tufano JJ, Kubový P, Jelen K. The effect of military boots on front kick dynamics. Acta Universitatis Carolinae: Kinanthropologica, 2018; 54(2):

Wasik J, Czarny W, Malolepszy E, Drozdek-Malolepsza T. Kinematics of taekwon-do front kick. Archives of Budo Science of Martial Arts and Extreme Sports, 2015; 11: 23-28

Wilk M, Petr M, Krzysztofik M, Zajac A, Stastny P. Endocrine response to high intensity barbell squats performed with constant movement tempo and variable training volume. Neuro Endocrinol Lett, 2018a; 39(4): 342-348

Wilk M, Stastny P, Golas A, Nawrocka M, Jelen K, Zajac A, Tufano JJ. Physiological responses to different neuromuscular movement task during eccentric bench press. Neuro Endocrinol Lett, 2018b; 39(1): 26-32

\section{Corresponding author:}

\section{doc. PhDr. Petr Stastny Ph.D.}

Charles University in Prague

Faculty of Physical Education and Sport,

Department of Sport Games

Jose Martiho 31

Prague 16252

Czech Republic

Phone: + 420 777198764,

E-mail: stastny@ftvs.cuni.cz 